\title{
Integro-Differential Non-Linear Equations and Continual Lie Algebras
}

\author{
M. V. Saveliev \\ Institute for High Energy Physics, SU-142284, Serpukhov, USSR
}

\begin{abstract}
The integrability problem of integro-differential equations with, generally speaking, singular kernels is discussed after an example of new continual analogs of the two-dimensional Toda lattices. These equations are associated with new infinite-dimensional Lie algebras via zero curvature type representation. The structural constants of these algebras are distributions. A formal solution of the Goursát problem is obtained. For the case with the kernel of the integral operator being $\delta_{ \pm}$-distribution an explicit expression in quadratures for the solutions is given.
\end{abstract}

\section{Introduction}

Recently an interest in the integrability problem for nonlinear integro-differential equations revived, mainly due to physicist-theoreticians and applied mathematicians. There would be no harm to note that this problem has been in a stagnant state for a long period. The renaissance in question is motivated, in particular, by the problems of fluid flow dynamics and plasma physics. Probably, it is not alien also to the gauge theories in particle physics. In this, the integro-differential equations such as Benjamin-Ono or sine-Hilbert are usually studied. These equations are derived from the wave or evolution differential equations by introducing in one of the terms an integral operator with the Cauchy or Hilbert kernel over a spatial argument (see, for example [1]). However, at present there are not any sufficiently general and self-consistent constructions of nonlinear integrodifferential equations and the integrability criteria for them.

Thus, it seems reasonable for this goal to try to generalize an algebraic approach which proved itself in a good light in the problem of constructing and explicit investigation of the integrable differential systems [2]. Here we take as the starting point in the spirit of the Volterra method [3] for transition from finite to infinite number of variables, a continual extension of the multicomponent differential systems. In the present paper the two-dimensional generalized Toda lattice is used as an example of such an initial differential system. Its continual 
extension, in which a new argument is a continuous limit of a lattice knot number, represents a new type of non-linear integro-differential equations. As far as I know this kind extension of the lattices and the corresponding equations were not earlier proposed.

Here there would be no harm to stress the following. First, the idea of considering the continuous analogs of the Toda lattice has been repeatedly discussed in literature (see, for example [4]). However, in all limits previously studied the systems are reduced to the equations of the Korteweg-de Vries type, i.e., they did not lead out of the framework of a pure differential picture. Second, the known equation for the induced Langmuir plasmon scattering by ions [5] just represents in the aforementioned sense the continual limit of the difference $\mathrm{KdV}$ (or, which is the same, the differential equations of Lotka-Volterra type). The latter serves as a Bäcklund transformation for the Toda lattice. Probably, the integrodifferential analogs of the discrete systems discussed here are not uninteresting for a series of physical applications.

As we will see below, an investigation of the problem of construction and integration of non-linear integro-differential equations in the spirit of the algebraic approach [2] requires the introduction of essentially new types of Lie algebras. For the latter ones the structural constants are the kernels of the integral, generally speaking, singular operators. By means of the Cartan-Maurer type equations these algebras generate the unknown non-linear equations. In this connection the integrals of motion and the characteristic integrals already become the non-local objects with respect to the corresponding argument. Their study uses the elements of singular integral equation theory, in particular, the formulas of PoincaréBertrand type.

For the continual generalizations of the Toda lattices one manages to explicitly construct a formal solution of the Goursát problem in the form of an infinite series. Establishing its convergence requires additional conditions to be put on the integral operator whose kernel is a continual analog of the Cartan matrices. Apparently, these conditions are related with the finiteness of functional growth of continual algebras in the spirit of Kolmogorov and Gel'fand. Such a conception should generalize the notion of finiteness of growth for the Kač-Moody algebras. In the simplest nontrivial case when the integral operator in question is the Hilbert operator, i.e., its kernel is the causal $\delta_{ \pm}$-function, the series is cut off on the second term. Then a solution of the corresponding integral generalization of the Liouville equation is given by a simple formula. For this kernel it is also possible to obtain the solutions of the integral generalization of the sine-Gordon equation on the class of $N$-soliton solutions.

The consideration and results of the present paper are partly announced in [6] and in the invited talk at the IX Congress of the IAMP (Swansea, U.K., July 1988).

\section{Derivation of Equation and Continual Lie Algebras}

Consider a two-dimensional generalized Toda lattice with fixed end-points described by the exactly integrable system of equations $[7,2]$

$$
\partial^{2} x_{i} / \partial z_{+} \partial z_{-}=\exp (k x)_{i}, \quad 1 \leqq i \leqq r .
$$


Here $k$ is the Cartan matrix of finite-dimensional simple Lie algebra $G$ over $\mathbb{C}$ of rank $r$. The proposed continual analog of this system has the form

$$
\partial^{2} x\left(z_{+}, z_{-} ; t\right) / \partial z_{+} \partial z_{-}=\exp \int_{L} d t^{\prime} K\left(t, t^{\prime}\right) x\left(z_{+}, z_{-} ; t^{\prime}\right) \equiv \exp (\hat{K} x),
$$

where $\hat{K}$ is an integral operator with, generally speaking, a singular kernel. The integral in (2) is taken over some contour $L$ and should be understood in the sense of the principal value. The new variable $t$ is just a continuous limit of a lattice index, i.e., $x_{i}\left(z_{+}, z_{-}\right) \rightarrow x\left(z_{+}, z_{-} ; t\right)$. The range of variation of $t$ and the contour in (2) are not fixed yet. In the simplest evident case when $K\left(t, t^{\prime}\right)=2 \delta\left(t-t^{\prime}\right)$, Eq. (2) reduces to the well-known Liouville equation, i.e., (1) with $r=1$ and $k=2$.

A continual analog of the periodic problem can be written in the same way,

$$
\partial^{2} x / \partial z_{+} \partial z_{-}=\exp (\hat{K} x)-\exp (-\hat{K} x) .
$$

It is interesting to compare Eq. (2) rewritten for the function $y \equiv \hat{K} x$, i.e.,

$$
\partial^{2} y(t) / \partial z_{+} \partial z_{-}=\int d t^{\prime} K\left(t, t^{\prime}\right) \exp y\left(t^{\prime}\right),
$$

with a structure of the aforementioned equation for the Langmuir waves with zero linear damping [5],

$$
\partial \varrho(k) / \partial \tau=\int d k^{\prime} T\left(k, k^{\prime}\right) \exp \varrho\left(k^{\prime}\right), \quad \varrho \equiv \ln n_{k}(\tau) .
$$

Before we begin with the integrability problem of the non-linear integrodifferential equations under consideration let us elucidate with which algebraic structures they are related. Remember for this aim that the algebraic approach for the integrability problem of two-dimensional differential systems [2] is based on the zero curvature representation

$$
\left[\partial / \partial z_{+}+A_{+}, \partial / \partial z_{-}+A_{-}\right]=0 .
$$

The latter generates the corresponding non-linear integrable equations by means of a realization of the functions $A_{ \pm}\left(z_{+}, z_{-}\right)$in the subspaces $\underset{m \geq 0}{\oplus} G_{ \pm m}$ of a graded Lie algebra $G=\bigoplus_{m \in \mathbb{Z}} G_{m}$ of finite growth, zero growth for exactly integrable systems as well. To carry out the analogous investigation of the integro-differential equations we need in the following a generalization of the algebraic structures. In this connection, as well as in the discrete case (1), the integrability of the equations is naturally defined by the properties of the operator $\hat{K}$ whose algebraic sense at first will be discussed by the example of Eq. (2).

One can easily be convinced that Eq. (2) is generated by representation (4) with the functions

$$
A_{ \pm}=\int d t\left[ \pm \frac{1}{2} h(t) \partial x(t) / \partial z_{ \pm}+X_{ \pm}(t) \exp \left(\frac{1}{2} \hat{K} x(t)\right)\right]
$$

where the elements $h(t)$ and $X_{ \pm}(t)$ obey the commutation relations

$\left[h(t), h\left(t^{\prime}\right)\right]=0, \quad\left[h(t), X_{ \pm}\left(t^{\prime}\right)\right]= \pm K\left(t^{\prime}, t\right) X_{ \pm}\left(t^{\prime}\right), \quad\left[X_{+}(t), X_{-}\left(t^{\prime}\right)\right]=\delta\left(t-t^{\prime}\right) h(t)$.

(Here and in what follows the dependence of the function $x$ on the arguments $z_{ \pm}$is omitted as a rule.) 
In analogy with the contragradient Lie algebras it is natural to consider these elements as the generators of a subspace (local part) $G_{(\ell)}$ of some infinitedimensional, in principle, Lie algebra $\hat{G}$. The latter as a whole is generated by the repeated commutations

$$
\left[X_{ \pm}\left(t_{n}\right)\left[X_{ \pm}\left(t_{n-1}\right) \ldots\left[X_{ \pm}\left(t_{2}\right), X_{ \pm}\left(t_{1}\right)\right] \ldots\right]\right]
$$

and the Jacobi identities for it are easily verified. The algebra $\hat{G}$ will be called the continual algebra. The function $K\left(t, t^{\prime}\right)$ is, generally speaking, a distribution and serves as a continual analog of the Cartan matrix. In the simplest singular case with $\delta$-type kernel the algebra $\hat{G}\left(=G_{(\ell)}\right)$ coincides with the centre-free current algebra without Schwinger term which, however, can be involved in the consideration.

The algebra $\hat{G}$ introduced above can be generalized if one changes $G_{(t)}$ by the subspace $G_{(\ell)}^{(R)}=\bigoplus_{m=0, \pm 1} G_{m}^{(R)}$ with the elements $X_{-\alpha}(t), h_{\alpha}(t)$ and $X_{+\alpha}(t), 1 \leqq \alpha \leqq R$, satisfying the relations

$$
\begin{gathered}
{\left[h_{\alpha}(t), h_{\beta}\left(t^{\prime}\right)\right]=0, \quad\left[h_{\alpha}(t), X_{ \pm \beta}\left(t^{\prime}\right)\right]= \pm K_{\beta \alpha}\left(t^{\prime}, t\right) X_{ \pm \beta}\left(t^{\prime}\right),} \\
{\left[X_{+\alpha}(t), X_{-\beta}\left(t^{\prime}\right)\right]=\delta_{\alpha \beta} \cdot \delta\left(t-t^{\prime}\right) h_{\alpha}(t) .}
\end{gathered}
$$

These elements generate the continual algebra $\hat{G}^{(R)}$ with the local part $G_{(\ell)}^{(R)}$. Then, realizing the functions $A_{ \pm}$in the subspaces $G_{0}^{(R)} \oplus G_{ \pm 1}^{(R)}$,

$$
A_{ \pm}=\int d t \sum_{\alpha=1}^{R}\left[ \pm \frac{1}{2} h_{\alpha}(t) \partial x_{\alpha}(t) / \partial z_{ \pm}+X_{ \pm \alpha}(t) \exp \left(\frac{1}{2} \hat{K} x(t)\right)_{\alpha}\right]
$$

we obtain from representation (4) the following multicomponent generalization of Eq. (2):

$$
\partial^{2} x_{\alpha}(t) / \partial z_{+} \partial z_{-}=\exp \int_{L} d t^{\prime} \sum_{\beta} K_{\alpha \beta}\left(t, t^{\prime}\right) x_{\beta}\left(t^{\prime}\right)
$$

The multicomponent generalization of Eq. (3) is found in the same manner.

\section{Solution of the Goursát Problem}

It is possible to obtain a formal solution of the Goursát problem for the equations under consideration with an arbitrary operator $\hat{K}$. This solution is represented in the form of an infinite series which is convergent only under the appropriate constraints on $\hat{K}$. We confine ourselves to consideration of Eq. (2).

For this goal supply the nonlinearity with a parameter $\lambda, \partial^{2} x / \partial z_{+} \partial z_{-}$ $=\lambda \exp (\hat{K} x)$, which one can put equal to 1 in the final expression for the solution. Expand $x(z, t ; \lambda)$ in a power series of $\lambda, x(z, t ; \lambda)=\sum_{n \geqq 0} \lambda^{n} x_{n}(z, t)$. Then the zero mode $x_{0}$ satisfies the Laplace equation, $\partial^{2} x_{0} / \partial z_{+} \hat{\partial} z_{-}=0$, so $x_{0}(z, t)=x_{0}^{+}\left(z_{+}, t\right)$ $+x_{0}^{-}\left(z_{-}, t\right)$. Performing a reccurrent procedure we obtain the expression

$$
\begin{aligned}
x(z, t ; \lambda)= & x_{0}(z, t)+\sum_{n \geqq 1} \lambda^{n} \int \ldots \int \prod_{m=1}^{n-1} d t_{m} \Phi^{+}\left(t, t_{1}, \ldots, t_{n-1}\right) \\
& \times \sum_{\omega} \Phi^{-}\left(t, t_{(\omega(1)}, \ldots, t_{(\omega(n-1)}\right)\left[K\left(t, t_{m}\right)+S_{m}\left(t_{1}, \ldots, t_{n-1} ; \omega\right)\right] .
\end{aligned}
$$


Here

$$
\begin{gathered}
\Phi^{ \pm}\left(t_{0}, t_{1}, \ldots, t_{n-1}\right) \equiv \int \ldots \int \prod_{m=1}^{n} d z_{m}^{ \pm} \theta\left(z_{m}^{ \pm}-1-z_{m}^{ \pm}\right) \exp y_{0}^{ \pm}\left(z_{m}^{ \pm}, t_{m-1}\right), \\
n \geqq 1, \quad z_{0}^{ \pm} \equiv z_{ \pm}, \quad t_{0} \equiv t ; \quad y_{0}^{ \pm} \equiv \hat{K} x_{0}^{ \pm} \\
S_{m} \equiv \sum_{\ell=1}^{m-1} K\left(t_{\ell}, t_{m}\right) \theta\left[\omega^{-1}(m)-\omega^{-1}(\ell)\right] ;
\end{gathered}
$$

$\theta(z)$ is the Heaviside function, $\omega$ is any permutation of the indices from 1 to $n-1$.

Note that expression (9) can be rewritten for the function $y \equiv \hat{K} x$ in a more symmetrical form with notations $\tau_{m} \equiv\left\{z_{m}^{+}, z_{m}^{-}, t_{m}\right\}, d \tau_{m} \equiv d z_{m}^{+} d z_{m}^{-} d t_{m}$, namely

$$
y(\tau ; \lambda)=y_{0}+\sum_{n \geqq 1} \lambda^{n} \int \ldots \int\left(\prod_{m=1}^{n} d \tau_{m} \exp y_{0}\left(\tau_{m}\right)\right) R_{n}\left(\tau, \tau_{1}, \ldots, \tau_{n}\right) .
$$

Here

$$
\begin{gathered}
R_{n} \equiv L\left(\tau, \tau_{1}\right) \prod_{m=1}^{n-1}\left[L\left(\tau_{m}, \tau_{m+1}\right)+(n-m+1)^{-1} \sum_{\ell=1}^{m-1} L\left(\tau_{\ell}, \tau_{m+1}\right)\right], \\
L\left(\tau_{\ell}, \tau_{m}\right)=\theta\left(z_{\ell}^{+}-z_{m}^{+}\right) \theta\left(z_{\ell}^{-}-z_{m}^{-}\right) K\left(t_{\ell}, t_{m}\right) .
\end{gathered}
$$

Using the multiplicative properties of $\Phi^{ \pm}$and putting $\lambda=1$, it is suitable to present (9) in a form

$$
\begin{aligned}
\exp [-x(z, t)]= & \exp \left[-x_{0}(z, t)\right] \\
& \times\left\{1+\sum_{n \geqq 1}(-1)^{n} \int \ldots \int \prod_{m=1}^{n-1} d t_{m} \Phi^{+}\left(t, t_{1}, \ldots, t_{n-1}\right)\right. \\
& \left.\times \sum_{\omega} \Phi^{-}\left(t, t_{\omega(1)}, \ldots, t_{\omega(n-1)}\right) D_{n}\left(t, t_{1}, \ldots, t_{n-1} ; \omega\right)\right\},
\end{aligned}
$$

where

$$
\begin{aligned}
& D_{n} \equiv \prod_{m=1}^{n-1}\left[\varepsilon_{\omega} \delta\left(t-t_{m}\right)-K\left(t, t_{m}\right)-S_{m}\left(t_{1}, \ldots, t_{n-1} ; \omega\right)\right] \text {, } \\
& \varepsilon_{\omega} \equiv\left\{\begin{array}{lll}
2 & \text { if } & S_{m}=0 \\
1 & & \neq 0
\end{array} .\right.
\end{aligned}
$$

This expression represents a direct continual extension of the known formula [2] for a two-dimensional generalized Toda lattice with fixed end-points associated with a finite-dimensional simple Lie algebra $G$. Due to the properties of the Cartan matrix of $G$ the number of terms in the series for the solutions $\exp \left[-x_{i}(z)\right]$ of the lattice is equal to the dimension $d_{i}$ of the $i^{\text {th }}$ fundamental representation of $G$, i.e., $D_{d_{t}+1}=0$. For the continual case (10) the picture becomes more sophisticated. Naturally, for $K\left(t, t^{\prime}\right)=2 \delta\left(t-t^{\prime}\right)$, i.e., when (2) reduces to the Liouville equation, $D_{2} \equiv 0$ and (10) reproduces the well-known general solution

$$
\exp \left[-x\left(z_{+}, z_{-}\right)\right]=\exp \left(-x_{0}\right)\left[1-\int_{a_{+}}^{z_{+}} d z_{+}^{\prime} e^{2 x_{0}^{+}\left(z^{\prime}\right)} \int_{a_{-}}^{z_{-}} d z_{-}^{\prime} e^{2 x_{0}^{-}\left(z^{\prime}\right)}\right] \text {. }
$$


However, already in the simplest nontrivial case when $K\left(t, t^{\prime}\right)$ is the Cauchy kernel, i.e., $\hat{K}=\hat{K}_{+} \equiv \hat{I}+i \hat{H}$ or $\hat{K}=\hat{K}_{-} \equiv \hat{I}-i \hat{H}, \hat{H}^{2}=-\hat{I}, K_{ \pm}\left(t, t^{\prime}\right) \equiv 2 \delta_{ \pm}\left(t-t^{\prime}\right)=\frac{i}{\pi}\left(t-t^{\prime}\right.$ $\pm i 0)^{-1}$ is the causal function, $\hat{I}$ is the identical operator, we have $D_{2}\left(t, t^{\prime}\right)=2 \delta\left(t-t^{\prime}\right)$ $-K_{ \pm}\left(t, t^{\prime}\right) \equiv K_{\mp}\left(t, t^{\prime}\right) \neq 0$. But in this

$$
\int d t^{\prime} D_{2}\left(t, t^{\prime}\right) \Phi^{+}\left(t, t^{\prime}\right) \Phi^{-}\left(t, t^{\prime}\right)=0
$$

due to the relation $\hat{K}_{ \pm} \exp \left(\hat{K}_{ \pm} x\right)=2 \exp \left(\hat{K}_{ \pm} x\right)$. That is why the general solution to Eq. (2) with such a kernel is given by the formula

$$
\exp \left[-x\left(z_{+}, z_{-} ; t\right)\right]=\exp \left(-x_{0}\right)\left[1-\int_{a_{-}}^{z_{+}} d z_{+}^{\prime} e^{\hat{K} x_{0}^{+}\left(z_{+}^{\prime}, t\right)} \int_{a_{-}}^{z_{-}} d z_{-}^{\prime} e^{\hat{K} x_{0}^{-}\left(z^{\prime}, t\right)}\right] \text {. }
$$

(The analogous construction takes place also for the Hilbert kernel.) In other words here a cut off in the series in (10) is provided not only by the properties of $\hat{K}$ but also by the properties of the "bare" function $\exp \left(\hat{K} x_{0}\right)$. This fact resembles the situation in the theory of dynamical symmetries where the "spectrum generating algebra" of the Schrödinger equation is closed for the appropriate potentials only on a class of its solutions. form

Similar to the case of the Toda lattices [2] expression (10) can be rewritten in a

$$
\begin{aligned}
\exp [ & -x(z, t)]=\exp \left[-x_{0}(z, t)\right] \cdot\left\langle t\left|M_{+}^{-1}\left(z_{+}\right) M_{-}\left(z_{-}\right)\right| t\right\rangle \\
= & \exp \left[-x_{0}(z, t)\right] \cdot\left\{1+\sum_{n \geqq 1}(-1)^{n} \int \ldots \int \prod_{m=1}^{n} d t_{m} d \tilde{t}_{m} \Phi^{+}\left(t_{1}, \ldots, t_{n}\right)\right. \\
& \left.\times \Phi^{-}\left(\tilde{t}_{1}, \ldots, \tilde{t}_{n}\right) \cdot\left\langle t\left|X_{+}\left(t_{1}\right) \ldots X_{+}\left(t_{n}\right) X_{-}\left(\tilde{t}_{n}\right) \ldots X_{-}\left(\tilde{t}_{1}\right)\right| t\right\rangle\right\} .
\end{aligned}
$$

Here $M_{ \pm}\left(z_{ \pm}\right)$are the solutions of the equations

$$
\partial M_{ \pm}\left(z_{ \pm}\right) / \partial z_{ \pm}=M_{ \pm}\left(z_{ \pm}\right) \int d t \exp y_{0}^{ \pm}\left(z_{ \pm}, t\right) X_{ \pm}(t),
$$

presented by the multiplicative integrals, $X_{ \pm}(t)$ are the elements of the continual algebra subjected to relations (6). In this relation

$$
\begin{aligned}
& \left\langle t\left|X_{+}\left(t_{1}\right) \ldots X_{+}\left(t_{n}\right) X_{-}\left(\tilde{t}_{n}\right) \ldots X_{-}\left(\tilde{t}_{1}\right)\right| t\right\rangle \\
& \quad=\delta\left(t-t_{1}\right) \delta\left(t-\tilde{t}_{1}\right) \sum_{\omega} \prod_{m=2}^{n} \delta\left(\tilde{t}_{m}-t_{\omega(m)}\right) D_{n}\left(t_{1}, \ldots, t_{n} ; \omega\right) ;
\end{aligned}
$$

$|t\rangle$ is a normalized basis vector for which $h\left(t^{\prime}\right)|t\rangle=\delta\left(t-t^{\prime}\right)|t\rangle, X_{+}\left(t^{\prime}\right)|t\rangle=0$ and $\langle t| X_{-}\left(t^{\prime}\right)=0$ for all $t^{\prime}$.

Expression (10) gives a formal solution of the Goursàt problem on the characteristics $z_{+}=a_{+}$. Investigation of the convergence properties of the infinite series in (10) is defined by the properties of the operator $\hat{K}$ or, in the end, by the continual algebra itself. For the case of Toda lattices we know [2] that the absolute convergence of the series for the periodic problem is provided by the finiteness of growth of the corresponding Kač-Moody algebra (for the fixed end-points of the lattice the algebra is finite-dimensional and this series converts into a finite polynomial). Here, for the continual algebras, we probably need a notion of 
finiteness of the functional growth. However, at present we do not have any satisfactory understanding of this question at our disposal. Thus in the next section we shall stick to some heuristic reasonings based on the notion of the characteristic integrals determined on a class of the solutions of Eq. (2).

\section{Characteristic Integrals}

Recall that for differential systems the presence of a complete set of the characteristic integrals bears witness on their integrability in a definite sense. At the same time, however, these integrals give a practical possibility to explicitly construct the solutions only for the simplest cases (see, for example [2]). To my knowledge, the problem in such a statement for the integro-differential equations has never been investigated. Here the characteristic integrals already become nonlocal objects that put obstacles in the way of their study.

In the second order with respect to the derivatives over $z_{+}$or $z_{-}$the expressions

$$
\begin{aligned}
& W_{2}^{ \pm}\left(\partial x / \partial z_{ \pm}, \partial^{2} x / \partial z_{ \pm}^{2}\right) \\
& \equiv \int d t v(t)\left\{\partial^{2} x(t) / \partial z_{ \pm}^{2}+C_{0}\left(\partial x(t) / \partial z_{ \pm}\right)^{2}\right. \\
& \left.\quad+\left(-\frac{1}{2}+C_{1}\right) \partial x(t) / \partial z_{ \pm} \hat{K} \partial x(t) / \partial z_{ \pm}+C_{2}\left(\hat{K} \partial x(t) / \partial z_{ \pm}\right)^{2}\right\}
\end{aligned}
$$

with $C_{m}=$ const, $0 \leqq m \leqq 2$, represent the characteristic integrals for Eq. (2). This means that $\partial W_{2}^{ \pm} / \partial z_{\mp}=0$ on a class of the solutions to Eq. (2) in the following cases.

The operator $\hat{K}$ should be either

(i) symmetrizable, i.e., there is such a function $v(t)$ that $v(t) K\left(t, t^{\prime}\right)=v\left(t^{\prime}\right) K\left(t^{\prime}, t\right)$; in this, $\sum_{m} C_{m} \hat{K}^{m}=0 ; C_{2}=0$ iff $C_{0}=C_{1}=0$, or

(ii) eigen-operator for the function $\exp (\hat{K} x)$, i.e., $\widehat{K} \exp (\widehat{K} x)=c \cdot \exp (\widehat{K} x)$, $c=$ const $\neq 0$; in this, $C_{0}=c(1+d) / 2, C_{1}=-d, C_{2}=d / 2 c ; d=$ const. Further on we put $c=2$ without waste of generality.

Note that if in the first case (i) the operator $\hat{K}$ is an invertible operator then it is the Lagrangian density which corresponds to Eq. (2),

$$
\mathscr{L}=\int d t v(t)\left[\frac{1}{2} \partial x(t) / \partial z_{+} \hat{K} \partial x(t) / \partial z_{-}+\exp (\hat{K} x(t))\right] .
$$

The higher order integrals for multicomponent systems of type (8), i.e., the functions

$$
W_{n}^{ \pm}\left(\partial x_{\alpha} / \partial z_{ \pm}, \partial^{2} x_{\alpha} / \partial z_{ \pm}^{2}, \ldots, \partial^{n} x_{\alpha} / \partial z_{ \pm}^{n}\right)
$$

for which $\partial W_{n}^{ \pm} / \partial z_{\mp}=0$ with account of Eq. (8), arise under the fulfillment of definite polynomial relations for the kernel.

Thus, the only thing we managed to obtain here for Eq. (2) is the singling out of two cases mentioned above. In this, the first one requires an additional specialization of the kernel $\widehat{K}$. The solution for the second case for $\widehat{K}=\widehat{K}_{ \pm}$follows, as we have already said in Sect. 3, from the formal solution of the Goursát problem constructed there. However, it seems useful to obtain this solution on the basis of the methods of the singular integral equation theory (see, for example [8]). 


\section{Solutions for the Integral Analogs of the Liouville and Sine-Gordon Equations}

Consider as the operator $\hat{K}$ in Eqs. (2) and (3) the operators $\hat{K}_{+}$or $\hat{K}_{-}$ whose kernels are $\delta_{ \pm}$-functions. Acting by them on these equations from the left with account of the relation $\hat{K} \exp y=2 \exp y, y \equiv \hat{K} x$, we obtain the Liouville and the sine-Gordon equations, $\partial^{2} y / \partial z_{+} \partial z_{-}=2 \exp y$ and $\partial^{2} y / \partial z_{+} \partial z_{-}$ $=2 \exp y-2 \exp (-y)$, respectively. As a result the problem of finding the solutions to Eqs. (2) and (3) for the operator under consideration reduces to the solution of the degenerated case of the singular characteristic integral equation

$$
\pm x(t)+\frac{1}{\pi i} \int_{L} d t^{\prime} \frac{x\left(t^{\prime}\right)}{t^{\prime}-t}= \pm y(t) .
$$

Here the inhomogeneity is the well-known general solution of the Liouville equation and $N$-soliton solution of the sine-Gordon equation. In other words, Eq. (15) determines the analytic $t$-dependence of arbitrary functions $x_{0}^{ \pm}\left(z_{ \pm}, t\right)$ of the solution to the Liouville equation (11) and of the soliton parameters of the sineGordon equation. In its turn Eq. (15) is solved by the standard methods of singular integral equation theory under maintenance of the corresponding Hölder type conditions. In particular, the final solution for the integral analog of the Liouville equation is given by formula (11).

Acknowledgements. The author had many useful discussions with O. I. Bogoyavlensky, A.A. Kirillov, I. M. Krichever, A. N. Leznov, Yu. I. Manin and A. V. Razumov, to whom I am deeply grateful.

\section{References}

1. Constantin, P., Lax, P.D., Majda, A.: Commun. Pure Appl. Math. 38, 715 (1985) Ablowitz, M.J., Fokas, A.S., Kruskal, M.D.: Phys. Lett. 120 A, 215 (1987)

2. Leznov, A.N., Saveliev, M.V.: Group methods for integration of non-linear dynamical systems. Moscow: Nauka 1985 (Birkhäuser-Verlag - to appear); Nonlinear equations and graded Lie algebras. J. Sov. Math. 36, 699--721 (1987); Exactly and completely integrable nonlinear dynamical systems. Preprint IHEP 87-195, Serpukhov 1987 (Acta Appl. Math. - to appear)

3. Volterra, V.: Theory of functionals, integral and integro-differential equations. New York: Dover Publ. 1958

4. Toda, M.: Suppl. Progr. Theor. Phys. 59, 1-161 (1976); Bogoyavlensky, O.I.: Izv. Akad. Nauk SSSR, Ser. Mat. 51, 737-784 (1987)

5. Zakharov, V.E., Musher, S.L., Rubenchik, A.M.: Phys. Rept. 129, 287-322 (1985)

6. Saveliev, M.V.: New continual analogs of two dimensional Toda lattices related with nonlinear integro-differential equations. Preprint IHEP 88-39, Serpukhov, 1988

7. Leznov, A.N., Saveliev, M.V.: Lett. Math. Phys. 3, 489-491 (1979)

8. Gakhov, F.D.: Boundary value problems, N.Y.: Pergamon 1966; Muskhelishvili, N.I.: Singular integral equations. Groningen: P. Noordhoff 1953

Communicated by H. Araki

Received July 19, 1988

Note added in proof. The same method as in Sect. 5 can be applied also to another wave and evolution integro-differential equations. For example, consider an integral analog of the $\mathrm{KdV}$ equation $x^{\prime}+\dddot{x} \pm i(x \hat{H} x)^{\circ}=0$. Its solutions is obtained via the solutions of Eq. (15) with the inhomogeneity being the $N$-soliton solution of the $\mathrm{KdV}$ equation $y^{\prime}+\dddot{y}+2 y \dot{y}=0$ with $y \equiv \hat{K}_{ \pm} x$. 